\title{
LIGNANS IN OLIVE STONES DISCARDED FROM THE OIL INDUSTRY. COMPARISON OF THREE EXTRACTION METHODS FOLLOWED BY HPLC-DAD-MS/MS AND ANTIOXIDANT CAPACITY DETERMINATION
}

\author{
JUAN PABLO INOSTROZA, JAVIERA TRONCOSO, CLAUDIA MARDONES, CAROLA VERGARA* \\ Department of Instrumental Analysis, Faculty of Pharmacy, University of Concepción, Concepción, Chile \\ Departamento de Análisis Instrumental, Facultad de Farmacia, Universidad de Concepción, Casilla 160-C, Concepción, Chile
}

\begin{abstract}
Lignans are secondary metabolites with diverse chemical structure, and are frequent in food grains. A lignan rich diet have a positive health impact over chronic diseases and protection against certain types of cancer. To enhance the intake of lignans the addition of this compound in processed food could be a solution, so the best approach is to find a source of lignans, especially one that is discarded as waste. In the olive oil production there is a large volume of phytotoxic waste generated, among these is the olive stone (OS), that it is generally burned for fuel. It has been reported the presence of lignans in olive stones extracted with methanol reflux.

Three extraction methods for lignans and polyphenolic compounds were assessed: solid-liquid ultrasound assisted extraction with basic hydrolysis, methanolic extraction with Soxhlet, and $\mathrm{CO}_{2}$ supercritical fluid extraction with methanol as a modifier. The determination of lignan content in OS samples were evaluated by HPLC with a core shell C18 column and the detection was in tandem with DAD and MS/MS. The extracts were also evaluated in their antioxidant capacity with ABTS, CUPRAC and ORAC assays and total polyphenol concentration.

The aim of this work is to evaluate different methods to extract lignans from OS discarded from the olive oil industry. The main lignan identified in OS extract was pinoresinol. The SFE method to extract pinoresinol was more efficient than Soxhlet and ultrasound assisted solid liquid extractions, producing a higher amount of pinoresinol in the extract in less time and with less amount of co-extracted interferents. The extract produced with Soxhlet had a higher antioxidant capacity.
\end{abstract}

Keywords: lignans, pinoresinol, olive stones, Soxhlet, supercritical fluid extraction, HPLC.

\section{INTRODUCTION}

The constant and steady consumption of lignan rich food have a positive impact over human health especially in chronic diseases, and protection against certain types of cancer [1]. These effects are attributed principally to the transformation of lignans in to enterolactone and enterodiol, called mammalian or enterolignans by microflora in the proximal colon [2]. Lignans are secondary metabolites with diverse chemical structure that mainly consist of phenol groups and aromatic rings. This compounds are more frequent in grains such as flaxseed or sesame, and in the bark of several types of trees such as olive trees or some Pinus species [3]. Lignans such as pinoresinol (PIN) and acetoxypinoresinol (AC-PIN) have been reported in the phenolic fraction of virgin olive oil $[4,5,6,7]$ and in olive mill wastewater [8].

In the olive oil production there is a large volume of waste generated by olive mills that can signify in an important environmental problem, due to the high concentration of phenols, lipids and organic acids that are phytotoxic [9]. Among the solid residues generated from this industry, the olive stone (OS), that makes between the 13 and $30 \%$ of the fruit weight, it is composed primarily from hemicellulose (24\%), cellulose (32\%) and lignin $(22 \%)$ and the main use for this waste is as fuel, production of activated carbon and resin, among others [10].

In four olive varieties three main lignans were identified; acetoxypinoresinol (AC-PIN), pinoresinol (PIN) and hydroxypinoresinol (OH-PIN). OliverasLopez et al [11] reported an average concentration of total lignan of $0.17 \mathrm{mg} / \mathrm{g}$ dried olive stones extracted with methanol reflux. Other authors have shown that in OS methanolic extracts the main phenolic compounds are secoiroids such as nuezhenide and its derivates [12].

To extract other phenolic compounds, such as lignans a more exhaustive or drastic methodology may be needed. There are difficulties in the lignan extraction in foods because of the high diversity in polarities which inevitably signifies that there will not be a single optimal conditions for all the main lignans. Among the different extraction methods for phenolic compounds from olive pomace there are most commonly used solid-liquid extraction with polar protic solvents or Soxhlet, however both of them involves a long extraction times [15].

The extraction of lignans assisted by the use of alkaline hydrolysis which can increase the lignan extraction yield from flaxseed by the rupture of links between lignans and other compounds via ester bonds $[14,15,16]$. The use of acid can lead to artefacts such as the formation of isolaricitin and anhydrosecoisolariciresinol [2]. To improve the extraction the use of ultrasound assistance could shorten the time necessary due to the aid that the cavitational force can provide to improve the extractability of the phenolic compounds [3].

Supercritical fluid extraction (SFE) with carbon dioxide $\left(\mathrm{CO}_{2}\right)$ is a technique that has been successfully used for the extraction of phenolic compounds. The use of SFE for lignan extraction have some advantages for low to medium polarity compounds from seeds, which can also be improved by the addition of methanol as modifier [17]. From olive leaves, using methanol as a modifier, high recoveries of tyrosol was achieved avoiding the coextraction of elevated quantities of other compounds [3].

Phenolic compounds obtained from natural sources with high antimicrobial, antitumor or antioxidant effects may have economic interest $[5,18]$ especially if they can be obtained from an unutilized waste. Mansour et al. [12] indicated that OS have potential as a source of phenolic compound due to the high antioxidant capacity of the extracts.

The aim of this work is to extract lignans from discarded olive stones and to evaluate the best method to produce an extract with interesting concentration of lignans and high antioxidant capacity.

\section{EXPERIMENTAL}

\section{Samples}

A large sample of solid olive mill waste was collected from the industry. The solids were air dried and the stones were separated from the skins and remnant pulp. The olive stones (OS) were washed with deionized water, dried and then grinded to a fine powder. The powdered sample was homogenized and divided into subsamples to evaluate the different extraction methods. The samples were kept at room temperature (average $20^{\circ} \mathrm{C}$ ) and protected from direct sun light until extraction. Once the samples were processed, the extracts were kept at $-20^{\circ} \mathrm{C}$ until analysis.

Reagents and standards

All solutions for high-performance liquid chromatography (HPLC) analyses were prepared in $18 \mathrm{M} \Omega$ deionized water. HPLC-grade acetonitrile, ethanol, cupric chloride dihydrate, ammonium acetate, calcium carbonate, formic acid, were obtained from Merck (Darmstadt, Germany). Pinoresinol, matairesinol, pinoresinol diglucoside, lariciresinol, hydroxymatairesinol, secoisolariciresinol standards were obtained from Phytolab (Vestenbergsgreuth, Germany). 6-hydroxy-2,5,7,8-tetramethylchroman-2-carboxylic acid (Trolox), neocuproine hemihydrate, 2,2'-azino-bis-3-ethylbenzthiazoline-6-sulphonic (ABTS), potassium persulfate, were purchased from the Sigma-Aldrich Chemical Co. (St. Louis, MO, USA). 


\section{Extraction methods}

The final extraction methods were employed after a multivariate optimization process at analytical scale in triplicate. Each extract was performed and analyzed in triplicate and all the results were corrected on dry weight basis.

\section{Soxhlet}

For the Soxhlet extraction the method from Oliveras-Lopez et al [11] was used with modification. In a $250 \mathrm{~mL}$ Soxhlet equipment $5 \mathrm{~g}$ of sample were placed in a cellulose thimble with hexane $(250 \mathrm{~mL})$ to eliminate any lipophilic compounds for $12 \mathrm{~h}$. The defatted sample was extracted with methanol in reflux for $24 \mathrm{~h}$ to ensure the highest content of extractable compounds in the final extract, in order to have a point of comparison for the other two methods. The collected extract was evaporated to eliminate the methanol and diluted up to $25 \mathrm{~mL}$ in mobile phase and filtered through a GV Durapore filter $(0.22 \mu \mathrm{m}$ pore size, $13 \mathrm{~mm}$ diameter, Millipore, Bedford, MA, USA) previous to HPLCDAD-MS/MS analysis

\section{Ultrasound assisted solid-liquid extraction with basic hydrolysis}

The solid-liquid ultrasound assisted extraction with basic hydrolysis (USB) was based in the previously described methods by Vergara et al. [19] and Alu'datt et al. [14] with modifications. To $2 \mathrm{~g}$ of sample were added $20 \mathrm{~mL}$ of $80 \%$ methanol with $\mathrm{NaOH} 1 \mathrm{~N}$ for the hydrolysis treatment. The sample was homogenized with an ultrasound probe in three 1 minute pulses at $65 \mathrm{~Hz}$ then it was left to macerate for 30 minutes, until a second round of ultrasound treatment. The sample was then centrifuged at $4000 \mathrm{rpm}$ for 5 minutes and the supernatant was collected and the exhausted solid was re-extracted under the same conditions. The methanol in the extracts was removed using a rotaevaporator at $35^{\circ} \mathrm{C}$ and then neutralized. A cleanup step was performed with a $\mathrm{C} 18 \mathrm{SPE}$ cartridge eluting with three volumes of methanol. The resulting extract was evaporated and adjusted to $25 \mathrm{~mL}$ with mobile phase and filtered through a GV Durapore filter $(0.22 \mu \mathrm{m}$ pore size, $13 \mathrm{~mm}$ diameter, Millipore, Bedford, MA, USA) before chromatographic analysis.

\section{Supercritical fluid extraction}

The optimized extraction was carried out with a $\mathrm{CO}$ supercritical fluid extractor Applied Separations, model Spe-ed ${ }^{\mathrm{TM}}$ SFE-2 (Allentown, PA, USA) in a temperature of $80{ }^{\circ} \mathrm{C}$ and $40 \mathrm{MPa}$ of pressure. Methanol $10 \%(\mathrm{v} / \mathrm{v})$ was used to modify the polarity of the extraction by means of a co-solvent pump. In each experiment the OS $(5 \mathrm{~g})$ was loaded into a $50 \mathrm{~mL}$ extraction cell and the remaining volume was filled with glass beads. The outlet valve was maintained at $120^{\circ} \mathrm{C}$ and the extraction time was $120 \mathrm{~min}$. The outflow was maintained at $5 \mathrm{~L} / \mathrm{min} \mathrm{CO}_{2}$. The extract was collected in $25 \mathrm{~mL}$ of methanol and diluted with mobile phase up to $25 \mathrm{~mL}$, and then stored until analysis at $-20{ }^{\circ} \mathrm{C}$ in amber bottles to protect them from light. Previous to chromatographic analysis the samples were filtered through a GV Durapore filter $(0.22 \mu \mathrm{m}$ pore size, $13 \mathrm{~mm}$ diameter, Millipore, Bedford, MA, USA).

\section{Analytical methods}

Determination of lignans by UHPLC-DAD-ESI-MS/MS in olive stone extracts

The lignans in the extracts were analyzed using a Shimadzu Nexera UHPLC/HPLC system (Kyoto, Japan), equipped with a quaternary LC-30AD pump, a DGU-20A5R degasser unit, a Prominence CTO-20 AC oven, a SIL30AC autosampler, and an SPD-M20A ultraviolet-visible (UV-vis) diode array detector (DAD) coupled in tandem with a liquid chromatography-tandem mass spectrometry (LC-MS/MS) detector (QTrap 3200, Applied Biosystems MDS Sciex, Dublin, CA, USA). Instrument control and data collection were performed using a Class-VP DAD Shimadzu Chromatography Data System and Analyst software for MS/MS analysis (Version 1.5.2, Shimadzu Co., Kyoto, Japan). The separation conditions using a Kinetex C18 core-shell column $(150 \times 4.6 \mathrm{~mm}, 2.6 \mu \mathrm{m})$ with a guard column (Phenomenex, Torrance, CA, USA) were at $25^{\circ} \mathrm{C}$, with a $50 \mu \mathrm{L}$ injection volume. The mobile phase gradient consisted of $0.1 \% \mathrm{v} / \mathrm{v}$ formic acid in water (solvent $\mathrm{A}$ ) and acetonitrile (solvent B). The flow rate was $0.4 \mathrm{~mL} / \mathrm{min}$. The gradient program was from 15 $\%$ to $45 \%$ solvent B in $42 \mathrm{~min}$, then raised and maintained at $100 \%$ for $3 \mathrm{~min}$, followed by 5 min of stabilization at $15.0 \% \mathrm{~B}$.

Electrospray ionization (ESI)-MS/MS was performed using negative ionization mode $(-3500 \mathrm{~V})$, drying temperature $350{ }^{\circ} \mathrm{C}$, nebulizer gas at 40 a.u., auxiliary gas at 20 a.u., and scanning range between $\mathrm{m} / \mathrm{z} 100$ and 1200 Compounds were identified by comparison of their retention times with those of available standards and analyzing their UV and MS/MS spectra. Quantifications using a DAD were performed at $280 \mathrm{~nm}$ using external calibration curves. All results were expressed in a dry matter basis.

\section{Total polyphenols and antioxidant capacity}

The antioxidant capacity and Total Polyphenol content of the OS extracts were performed in 96-well microplates, using a micro-volume spectrophotometer (Epoch Biotek System, Winooski, VT, USA). Standard solutions were prepared mixed and individually, at the same concentration level found in the extract with the objective to compare the antioxidant capacities present in the extracts obtained with the different methods. The samples and standards were diluted in order to fit the linear range and were analyzed by triplicated.

Total Polyphenol: $50 \mu \mathrm{L}$ of sample were mixed in the 96-well microplate with $50 \mu \mathrm{L}$ Folin Ciocalteu reactive and $100 \mu \mathrm{L}$ of $6 \%$ sodium carbonate. The microplate was kept in the dark at room temperature for $2 \mathrm{~h}$. The absorbance of samples and calibration standards were measured at $760 \mathrm{~nm}$ in triplicated. The reagents in the same proportion were used as blank and gallic acid was used to calibrate.

ABTS assay: the 2,2'-azino-bis-3-ethylbenzthiazoline-6-sulphonic acid radical $\left(\mathrm{ABTS}^{\cdot+}\right.$ ) was prepared by mixing $7 \mathrm{mM} \mathrm{ABTS}$ and $2.45 \mathrm{mM} \mathrm{K} \mathrm{S}_{2} \mathrm{O}_{8}$ in deionized water for $16 \mathrm{~h}$ under darkness. This solution was diluted to obtain an absorbance of 0.7 at $734 \mathrm{~nm}$. The diluted solution $(170 \mu \mathrm{L})$ was mixed with Trolox or sample $(30 \mu \mathrm{L})$ for $20 \mathrm{~min}$ at $30^{\circ} \mathrm{C}$ and the absorbance was measured at the same wavelength. The antioxidant capacity was calculated as the Trolox Equivalent Antioxidant Capacity (TEAC) difference between blank and sample measurement, expressed as Trolox equivalents and considering the dilution factor [20].

Cupric ion reducing antioxidant capacity assay (CUPRAC): A mixture of $10 \mathrm{mM}$ copper(II), $7.5 \mathrm{mM}$ neocuproine, and ammonium acetate buffer $(\mathrm{pH} 7)$ was incubated at $37^{\circ} \mathrm{C}$ for $15 \mathrm{~min}$. Trolox or sample $(100 \mu \mathrm{L})$ was added and the absorbance was measured at $450 \mathrm{~nm}$ after incubation for $30 \mathrm{~min}$ at $37^{\circ} \mathrm{C}$ (solution 1). A sample prepared without copper (II) and neocuproine was used to correct the intrinsic absorbance of the sample (solution 2). The antioxidan activity was calculated by measuring the increase in the corrected absorbance (Asol1 - Asol2). The results were expressed as Trolox equivalents [21].

Oxygen radical absorbance capacity - fluorescein assay (ORAC-FL): were performed as a modification of the method detailed by Ou et al. [22]. Analyses were conducted in $\mathrm{pH} 7.0$ phosphate buffer $\left(\mathrm{K}_{2} \mathrm{HPO}_{4} 75 \mathrm{mM}\right.$ and $\mathrm{KH}_{2} \mathrm{PO}_{4} 75 \mathrm{~m}$ $\mathrm{M})$ at $37^{\circ} \mathrm{C}$. Peroxyl radical was generated using $25 \mu \mathrm{L}$ of $\mathrm{AAPH} 152 \mathrm{mM}$, and $150 \mu \mathrm{L}$ of fluorescein $0.011 \mu \mathrm{M}$ was used as the substrate. Samples and standards $(25 \mu \mathrm{L})$ were mixed with the reaction solution for $30 \mathrm{~min}$. Trolox was used as a standard and the fluorescence conditions of detection were $485 \mathrm{~nm}$ for excitation and $528 \mathrm{~nm}$ for emission.

\section{RESULTS AND DISCUSSION}

\section{Analytical methods for determination of lignans in OS extracts}

The identification of main lignans present in an OS extract was performed by comparison of the retention times and UV and MS spectra of the main lignans screened in the samples (figure 1). The fragmentation patterns are consistent with those previously reported in literature and there were confirmed by comparison with the available commercial standards.

In order to explore for the presence of another compounds found in the extracts a two experiment analysis was performed in the mass spectrometer. First a full scan mode analysis using the ion trap (EMS) was linked with a product ion analysis (EPI) of the highest mass found in the first experiment (EMS-IDA-EPI). In all of the OS extracts, pinoresinol was the main lignan identified. The analytical properties for the chromatographic analysis are summarized in table 1 . 


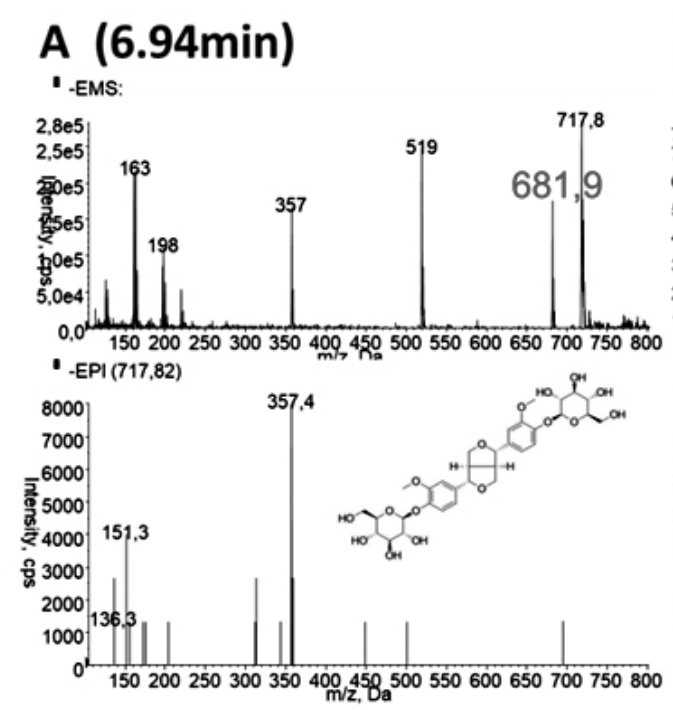

B $(\mathbf{1 5}, \mathbf{3 5 m i n})$
-EMS:

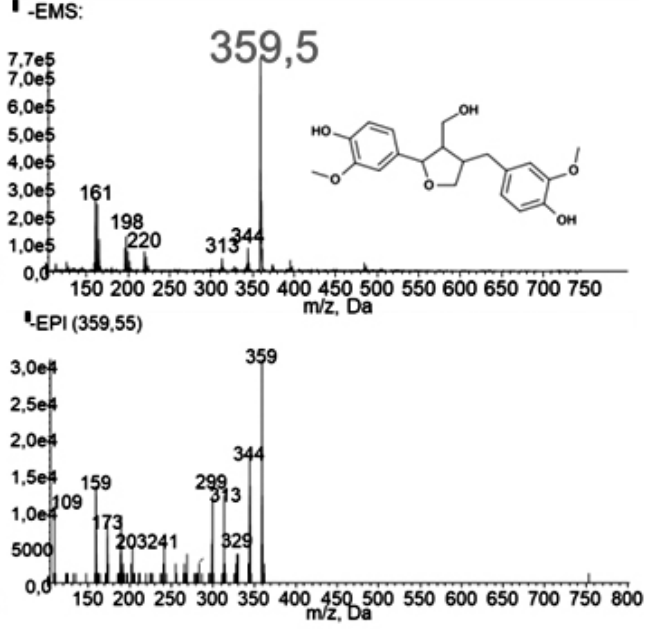

\section{$C_{1}(19.06 \mathrm{~min})$}

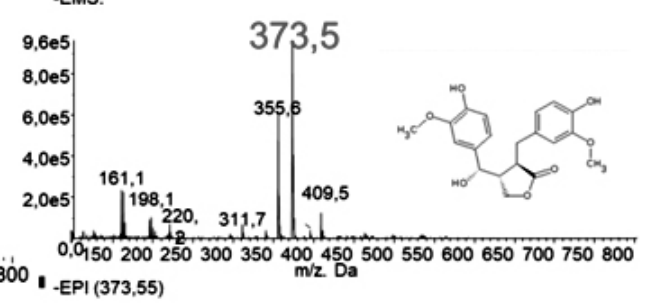

D (20.05 min)
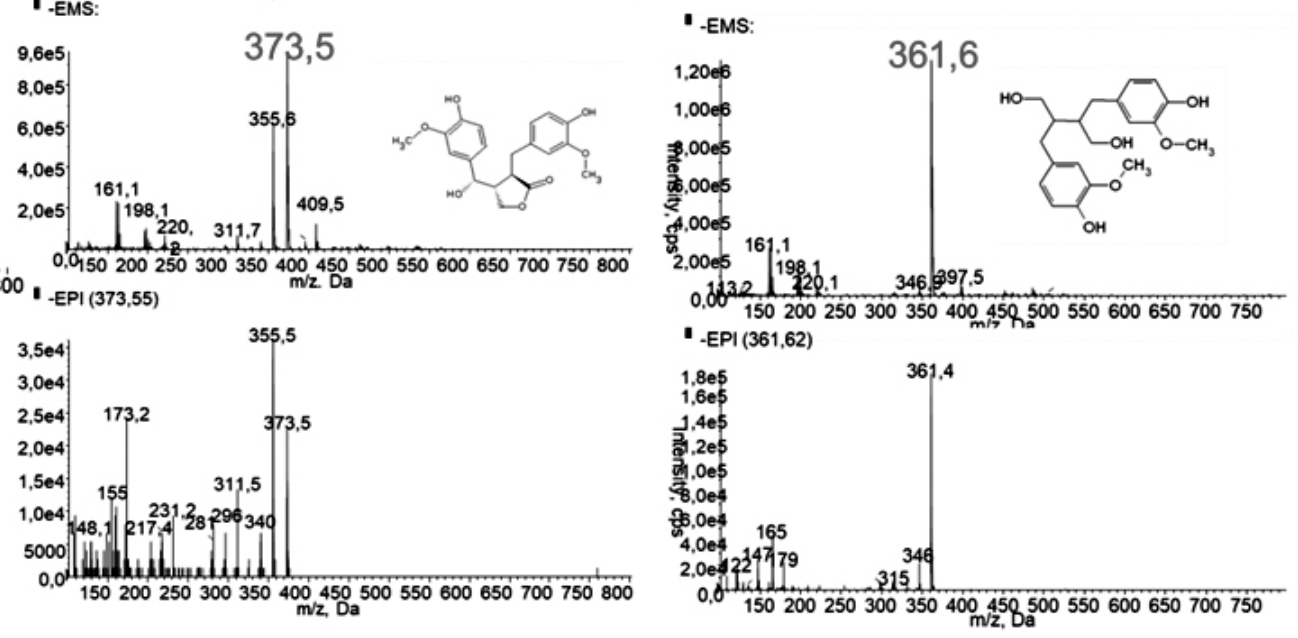

\section{F (34,35min)}

1 -EMS:

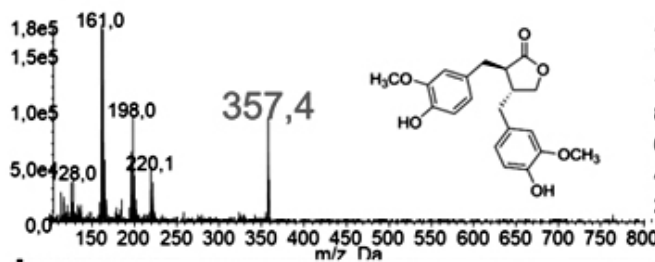

I -EPI $(357,46)$
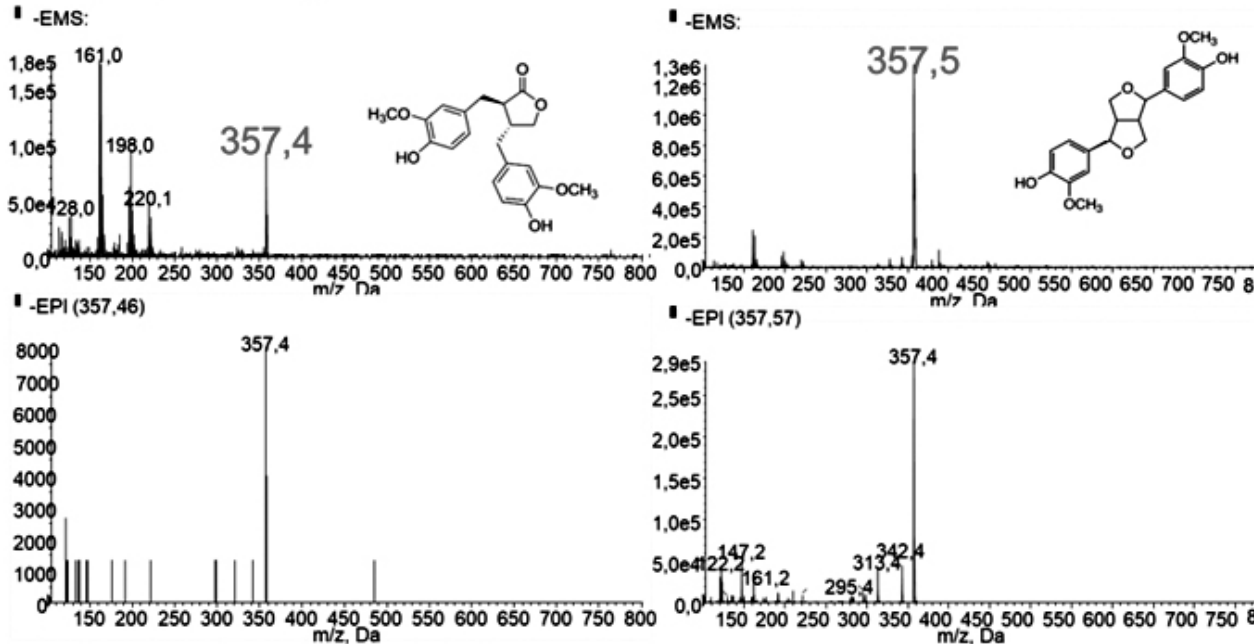

$0,0150200250300350400 \quad 450500550600650700750800$
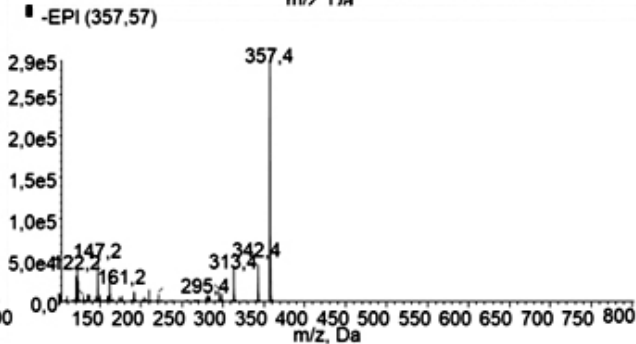

Figure 1: MS/MS spectra of targeted lignans screened in OS samples. Identification: A, pinoresinol diglucoside; B, lariciresinol; C, hydroxymatairesinol; D, secoisolariciresinol; E, pinoresinol; F, matairesinol. 
Table 1: Analytical parameters for used method for the analysis of OS extracts.

\begin{tabular}{|c|c|c|c|c|c|}
\hline & Equation & $\mathrm{R}^{2}$ & Typical error & LOD & LOQ \\
\hline $\begin{array}{l}\text { Total polyphenols } \\
\text { (Folin-Cicalteu) }\end{array}$ & $y=0.0268 x+0.0274$ & 0.989 & 0.01748 & $12 \mathrm{mg} / \mathrm{L}$ & $40 \mathrm{mg} / \mathrm{L}$ \\
\hline $\begin{array}{c}\text { TEAC } \\
\text { (CUPRAC) }\end{array}$ & $y=0.0034 x-0.0007$ & 0.9998 & 0.00252 & $2.2 \mu \mathrm{M}$ & $7.4 \mu \mathrm{M}$ \\
\hline $\begin{array}{l}\text { TEAC } \\
\text { (ABTS) }\end{array}$ & $y=0.0268 x+0.0878$ & 0.9729 & 0.018997 & $2.1 \mu \mathrm{M}$ & $7.1 \mu \mathrm{M}$ \\
\hline $\begin{array}{l}\text { TEAC } \\
(\mathrm{ORAC})\end{array}$ & $y=1.6774 x-2.107$ & 0.994 & 7.092 & $13 \mu \mathrm{M}$ & $42 \mu \mathrm{M}$ \\
\hline
\end{tabular}

Lignan extraction

To compare the extractions methods, the PIN concentration was evaluated alongside of the total polyphenolic content obtained in the extracts. These concentrations were calculated in a dry matter base for the sample and the final extract.

Comparing the chromatograms of the extracts of OS (figure 2) it can be observed that with the Soxhlet and US-B extractions there is a higher amount of compounds co-extracted making very difficult the determination of the targeted compounds. The extraction with SFE was considerably improved by the use of methanol as a co-solvent, where a larger amount of the targeted compound was achieved a cleaner separation was achieved, and the identification y subsequent quantification of pinoresinol was greatly improved.

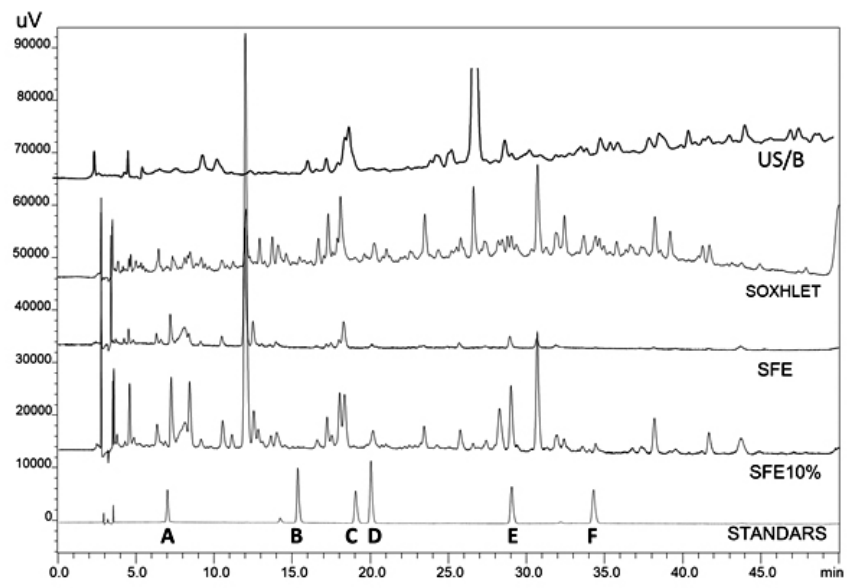

Figure 2: chromatograms of OS extracts made with (US/B) ultrasound assisted solid liquid extraction with basic hydrolysis; Soxhlet extraction; (SFE) $\mathrm{CO}_{2}$ supercritical fluid extraction; (SFE10\%) $\mathrm{CO}_{2}$ supercritical fluid extraction with $10 \%$ methanol. Standards identification in figure 1 .

Table 2: Pinoresinol concentration in olive stones by three extractive methodologies

\begin{tabular}{|c|c|c|c|c|c|}
\hline \multirow[b]{2}{*}{ Method } & \multirow{2}{*}{$\begin{array}{l}\text { Total } \\
\text { extraction } \\
\text { time } \\
\text { (h) }\end{array}$} & \multicolumn{2}{|c|}{ Pinoresinol } & \multicolumn{2}{|c|}{ Total Polyphenols } \\
\hline & & (mg/100g DW OS) & $\begin{array}{l}(\mathrm{mg} / 100 \mathrm{~g} \mathrm{DW} \\
\text { extract) }\end{array}$ & $\begin{array}{c}(\mathrm{GAE} m g / 100 \mathrm{~g} \mathrm{DW} \\
\mathrm{OS})\end{array}$ & $\begin{array}{c}\text { (GAE g/100g DW } \\
\text { extract) }\end{array}$ \\
\hline US-B & 4 & $4.14 \pm 0.01$ & $196.4 \pm 1.2$ & $0.95 \pm 0.01$ & $4.4 \pm 0.38$ \\
\hline SFE $10 \%$ methanol & 2 & $1.01 \pm 0.10$ & $308.3 \pm 2.8$ & $0.08 \pm 0.01$ & $2.4 \pm 1.21$ \\
\hline
\end{tabular}

Note: DW: dry weight; $\mathrm{n}=3$; GAE: gallic acid equivalent.

Comparing the pinoresinol concentration obtained with the three extraction methods showed that the highest concentration of PIN was obtained through the extraction US-B, with twice as much than PIN extracted with Soxhlet. And with SFE $+10 \%$ methanol half of the PIN extracted by Soxhlet was achieved (Table 2). If this comparison is made in basis of the concentration of PIN is expressed as the final dry extract obtained, the figure is reverted. The highest concentration was reached with the SFE $+10 \%$ methanol and the lowest was with the Soxhlet extraction. This is consistent with the observed in the chromatograms of figure 2 were the number of compounds coextracted with Soxhlet is considerably higher leading to a diminution in the proportion of PIN in the final extract. If this is compared with the total polyphenols content extracted on the basis of the dry matrix or in the final extract the same figure can be observed. With the SFE extraction a higher proportion of PIN is extracted with less co-extraction of other compounds. The total extraction time indicated in table 2 considers the complete sample treatment. The actual extraction time was determined during the optimization process and set at a 
point where an increase in time would not have a statistically significant increase in total lignan concentration. So, increasing the time for by US-B or SFE $+10 \%$ methanol extractions would result in an extract with the same quantity of target compounds, but maybe with a larger amount of co-extracted compounds that may affect the purity of a final extract.

Oliveras-Lopez et al. [11] reported concentration of PIN between 0.016 and $0.037 \mathrm{mg} / \mathrm{g}$ of dry stones in four olive varieties obtained with methanol at reflux, with an average of $2.7 \mathrm{mg} / 100 \mathrm{~g}$ of OS, which is three times as much as the PIN extracted with the SFE extraction $(1.01 \pm 0.03 \mathrm{mg} / 100 \mathrm{~g}$ of dry OS), but with a method that required more than $24 \mathrm{~h}$. It must be considered that in the first work, the olive stones were procured directly from fresh olive drupes, where in this case the olive stones are collected from the solid waste of the olive mill after the oils have been already extracted. With SFE the amount of total polyphenols extracted was $7.9 \pm 0.1 \mathrm{mg}$ of GAE/ $100 \mathrm{~g}$ of dry OS and 2.4 $\mathrm{g}$ GAE/ g of dry extract. In Moroccan varieties of olives the stones have been reported to have a total polyphenol, content between 3.6 and $11.3 \mathrm{~g} \mathrm{GAE} / \mathrm{g}$ of dry weight with Soxhlet and between 4.0 and $11.5 \mathrm{~g} \mathrm{GAE} / \mathrm{g}$ with ultrasound assistance from fresh olive drupes [23].

Considering the time necessary and the concentration achieved the SFE extraction method is the most efficient to produce a more concentrated PIN extract of all the assayed methods, with a maximum amount of PIN obtained of $0.3 \%$ in the final extract.

\section{Antioxidant capacity of OS extracts}

The analytical parameters for the antioxidant capacity of the extracts are presented in table 1 .

From these assays it can be observed that the extract with the highest antioxidant capacity is the one obtained with Soxhlet. In table 3 are summarized the results for the antioxidant capacity of olive stones extracts normalized to $\mathrm{Kg}$ of sample and to $100 \mathrm{~g}$ of produced extract. There is no doubt that by Soxhlet there are a larger amount of polyphenolic compounds extracted from the sample, which can be observed in the Folin-Ciocalteu assay and that are most likely other type of polyphenols with antioxidant capacity that have been already described $[11,12]$, this also is shown by the higher antioxidant capacity measured by ABTS, CUPRAC and ORAC-FL assays. However, this pattern is reverted when the antioxidant capacity is considered in function of the extract produced, where the higher levels were found in the extract produced by SFE, especially since this extract has a lower concentration of polyphenols than the extract made by Soxhlet, and a higher concentration of pinoresinol. In an OS extract made by solid-liquid extraction with $80 \%$ methanol an antioxidant activity by ABTS assay was evaluated reaching a TEAC of $436 \mu \mathrm{M}$ [12], which compared with the liquid extracts produced by US-B, Soxhlet and SFE were $389 \pm 13 \mu \mathrm{M}, 3369 \pm 158 \mu \mathrm{M}$ and $2391 \pm 347 \mu \mathrm{M}$, respectively, it can be compared to the solid-liquid extraction assisted with ultrasound, but considerable lower than the Soxhlet and SFE extraction methods.

Table 3: Antioxidant capacity in extracted OS samples by three methods.

\begin{tabular}{|c|c|c|c|c|c|c|}
\hline & \multicolumn{2}{|c|}{ ABTS } & \multicolumn{2}{|c|}{ CUPRAC } & \multicolumn{2}{|c|}{ ORAC } \\
\hline & $\begin{array}{c}\text { TEAC mmol } / \mathrm{kg} \\
\text { DW OS }\end{array}$ & $\begin{array}{c}\text { TEAC mol } / 100 \mathrm{~g} \\
\text { DW extract) }\end{array}$ & $\begin{array}{c}\text { mmol TEAC } / \mathrm{kg} \\
\text { DW OS }\end{array}$ & $\begin{array}{c}\text { TEAC mol } / 100 \mathrm{~g} \\
\text { DW extract) }\end{array}$ & $\begin{array}{c}\text { mmol TEAC } / \mathrm{kg} \\
\text { DW OS }\end{array}$ & $\begin{array}{c}\text { TEAC mol } / 100 \mathrm{~g} \\
\text { DW extract) }\end{array}$ \\
\hline US-B & $4.9 \pm 0.2$ & $28.9 \pm 0.2$ & $1.1 \pm 0.2$ & $0.5 \pm 0.1$ & $11.8 \pm 0.3$ & $121.6 \pm 0.4$ \\
\hline Soxhlet & $16.8 \pm 0.8$ & $6.2 \pm 0.2$ & $29 \pm 2$ & $133 \pm 1$ & $342 \pm 25$ & $461.4 \pm 0.2$ \\
\hline $\begin{array}{l}\text { SFE } 10 \% \\
\text { methanol }\end{array}$ & $11.9 \pm 1.7$ & $25.84 \pm 0.6$ & $17 \pm 2$ & $439.8 \pm 2.3$ & $127 \pm 15$ & $1602 \pm 7$ \\
\hline
\end{tabular}

Note: DW: dry weight; $n=3$; TEAC: Trolox equivalent antioxidant capacity.

\section{CONCLUSION}

The main lignan identified in olive stone extract was pinoresinol. The ultrasound solid liquid extraction assisted by basic hydrolysis was the method with the highest amount of PIN extracted, however with SFE using $10 \%$ of methanol produced the highest concentration of PIN in the final extract. With Soxhlet, extracts with higher antioxidant capacity were produced per $\mathrm{Kg}$ of sample, but this capacity was higher in the dry extracts produced with SFE.

The SFE method to extract a valuable lignan such as pinoresinol was more efficient than Soxhlet and ultrasound assisted solid liquid extractions, producing a higher amount of PIN in the extract in less time and with less amount of interferents co-extracted.

\section{ACKNOWLEDGMENTS}

The authors want to acknowledge the financial support of FONDECYT 11140229 and Proyecto PFB-27 grants.

\section{REFERENCES}

1. N. Pellegrini, S. Valtuena, D. Ardigo, F. Brighenti, L. Franzini, D. Del Rio, F. Scazzina, P.M. Piatti, I. Zavaroni, Nutr. Metab. Cardiovasc. Dis. $20,64,(2010)$

2. H. Adlercreutz, Crit. Rev. Clin. Lab. Sci. 44, 483, (2007)

3. E. Roselló-Soto, M. Koubaa, A. Moubarik, R.P. Lopes, J.A Saraiva, N. Boussetta, N. Grimi, F.J. Barba, Trends Food Sci Technol. 45, 296, (2015)

4. E. Antonini, A. Farina, E.S. Scarpa, A. Frati, P.Ninfali, Int J Food Sci Nutr, 67, 9, (2016)

5. A. López-Biedma, C. Sánchez-Quesada, M. Delgado-Rodríguez, J.J. Gaforio, J Funct Foods. 26, 36, (2016)

6. H.K. Obied, D.R. Bedgood, P.D. Prenzler, K. Robards, Anal. Chim, Acta, 603, 176, (2007)

7. R.W. Owen, W. Mier, A. Giacosa, W.E.Hull, B. Spiegelhalder, H. Bartsch, Clin. Chem. 46(7), 976, (2000)
8. J. Poerschmann, B. Weiner, I. Baskyr, Chemosphere, 92, 1472, (2013)

9. A. Roig, M.L. Cayuela, M.A. Sanchez-Monedero, Waste Manag., 26, 960, (2006)

10. R. Ghanbari, F. Anwar, K.M. Alkharfy, A.H. Gilani, N. Saari, Int. J. Mol. Sci. 13, 3291, (2012)

11. M.J. Oliveras López, M. Innocenti, F. Ieri, C. Giaccherini, A. Romani, N Mulinacci, J. Food Comp. Anal. 21, 62, (2008)

12. A.B. Mansour, E.A. Porter, G.C. Kite, M.S.J. Simmonds, R. Abdelhedi, M. Bouaziz, J. Agric. Food Chem. 63, 1990, (2015)

13. D. Boskou. Olive and Olive Oil Bioactive Constituents. Academic Press and AOCS Press, 2015.

14. M.H. Alu'datt, I. Alli, K. Ereifej, M. Alhamad, A.R. Al-Tawaha, T. Rababah. Food Chem. 123, 117, (2010)

15. I.E.J. Milder, I.C.W. Arts, D.P. Venema, J.J.P. Lasaroms, K.Wähälä, P.C.H. Hollman, J. Agric. Food Chem. 52, 4643, (2004)

16. I.E.J. Milder, I.C.W. Arts, B. van de Putte, D.P. Venema, P.C.H. Hollman, Br. J. Nutr. 93, 393, (2005)

17. S.M. Willfor, A.I. Smeds, B.R. Holmbom. J. Chromat. A, 1112, 64, (2006)

18. M. Araújo, F.B. Pimentel, R.C. Alves, M.B.P.P. Oliveira, Trends Food Sci. Technol. 45, 200, (2015)

19. C. Vergara, D. von Baer, C. Mardones, A. Wilkens, K. Wernekinck, A. Damm, S. Macke, T. Gorena, P. Winterhalter, J Agric Food Chem. 60 , 929, (2012)

20. C.N. Lin, A.M. Huang, K.W. Lin, T.C. Hour, H.H. Ko, S.C. Yang, Y.S $\mathrm{Pu}$, Phytochem. 71, 2140, (2010)

21. J.P.N. Ribeiro, L.M. Magalhães, S. Reis, J.L.F.C. Lima, M. A. Segundo, Anal. Sci. 27, 483, (2011)

22. B. Ou, H. Dejian, M. Hampsch-Woodill, J.A. Flanagan, E. K. Deemer, J. Agric. Food Chem. 50(11), 3122, (2002)

23. M. Elbir, N.E. Es-Safi, A. Amhoud, M. Mbarki, Maderas Cienc Tecnol 17(3), 479, (2015) 